\title{
Fetal exposure to the maternal microbiota in humans and mice
}

\author{
Noelle Younge, ${ }^{1}$ Jessica R. McCann, ${ }^{1}$ Julie Ballard, ${ }^{1,2}$ Catherine Plunkett, ${ }^{2}$ Suhail Akhtar, ${ }^{2}$ \\ Félix Araújo-Pérez,, Amy Murtha, ${ }^{1,3}$ Debra Brandon, ${ }^{1,4}$ and Patrick C. Seed ${ }^{2}$ \\ 'Department of Pediatrics, Duke University School of Medicine, Durham, North Carolina, USA. ${ }^{2}$ Department of Pediatrics, \\ Northwestern University, Chicago, Illinois, USA. ${ }^{3}$ Department of Obstetrics, Gynecology, and Reproductive Sciences, UCSF, \\ California, USA. ${ }^{4}$ School of Nursing, Duke University, Durham, North Carolina, USA.
}

Previous studies have demonstrated the presence of microbial DNA in the fetal environment. However, it remains unclear whether this DNA represents viable bacteria and how it relates to the maternal microbiota across body sites. We studied the microbiota of human and mouse dyads to understand these relationships, localize bacteria in the fetus, and demonstrate bacterial viability. In human preterm and full-term mother-infant dyads at the time of cesarean delivery, the oral cavity and meconium of newborn infants born as early as $\mathbf{2 4}$ weeks of gestation contained a microbiota that was predicted to originate from in utero sources, including the placenta. Using operative deliveries of pregnant mice under highly controlled, sterile conditions in the laboratory, composition, visualization, and viability of bacteria in the in utero compartment and fetal intestine were demonstrated by 165 rRNA gene sequencing, fluorescence in situ hybridization, and bacterial culture. The composition and predicted source of the fetal gut microbiota shifted between mid- and late gestation. Cultivatable bacteria in the fetal intestine were found during mid-gestation but not late gestation. Our results demonstrate a dynamic, viable mammalian fetal microbiota during in utero development.

Conflict of interest: The authors have declared that no conflict of interest exists.

Copyright: () 2019, American Society for Clinical Investigation.

Submitted: March 19, 2019

Accepted: August 27, 2019

Published: October 3, 2019

Reference information: /CI Insight. 2019;4(19):e127806.

https://doi.org/10.1172/jci.

insight.127806.

\section{Introduction}

Microbial colonization of the skin and mucosal surfaces in early life is essential for the development of host immunity and metabolism (1-3). The leading paradigm of early life colonization posits that the fetus develops in a sterile in utero environment and becomes colonized with microbes after birth through the process of vaginal delivery, mother's own human milk feeding, and other environmental exposures (4-6). However, next-generation sequencing has identified DNA from diverse human-associated bacteria in the human placenta, amniotic fluid, and infant meconium, suggesting that the first encounters with microbes may occur prenatally, even in the setting of a healthy pregnancy (7-13). These findings have generated new hypotheses for the maternal reservoirs of these bacteria and their effects on fetal development. One such hypothesis, based on studies demonstrating commonalities between the maternal and fetal microbiota, is that microbes in the maternal gut or oral cavity may be trafficked to the materno-fetal barrier hematogenously or via immune cells $(7,8,14,15)$. However, the timing and nature of fetal microbial exposures remain largely unknown. Important questions remain as to whether these bacterial genes represent viable and metabolically active bacteria, their source and route of passage to the fetus, and whether they persist after birth to shape the early neonatal microbiome.

Determining the presence and composition of the fetal microbiome has been challenging given technical and ethical constraints, including the noncultivatable nature of many organisms, the risks of invasive testing of the human fetus, and the challenge of discerning a fetal microbiota from ubiquitous environmental contaminants (16). If microbial DNA is indeed present in the fetal environment in the absence of pathologic infection, current data support that it is of low abundance $(17,18)$. The interpretation of bacterial DNA sequencing data in low-bacterial biomass environments is commonly confounded by background contamination (19). Although some studies have failed to identify a host-associated microbiota distinct from contaminating DNA $(16,20-22)$, other studies support the presence of unique microbial DNA in the fetal environment beyond background contamination $(17,18,23,24)$. Given the technical limitations of sequencing in low-bacterial biomass samples, multiple complementary approaches, including cultivation 
Table 1. Clinical characteristics of human mother-infant dyads

\begin{tabular}{|c|c|c|c|c|c|c|}
\hline Subject & $\begin{array}{l}\text { Birth weight } \\
\text { (grams) }\end{array}$ & $\begin{array}{l}\text { Birth gestational age } \\
\text { (weeks) }\end{array}$ & Sex & Labor before delivery & $\begin{array}{c}\text { Rupture of membranes } \\
\text { before delivery }\end{array}$ & Delivery indication \\
\hline \multicolumn{7}{|l|}{ Preterm } \\
\hline PT1 & 780 & 27 & $\mathrm{~F}$ & $\mathrm{~N}$ & $\mathrm{~N}$ & Preeclampsia \\
\hline РT2 & 640 & 26 & $\mathrm{~F}$ & $\mathrm{~N}$ & $\mathrm{~N}$ & Preeclampsia \\
\hline РT3 & 780 & 25 & $\mathrm{~F}$ & $\mathrm{Y}$ & $\mathrm{Y}$ & Preterm labor \\
\hline PT4 & 680 & 29 & $M$ & $\mathrm{~N}$ & $\mathrm{~N}$ & Fetal status ${ }^{A}$ \\
\hline PT5 & 640 & 24 & $\mathrm{~F}$ & $\mathrm{Y}$ & $\mathrm{N}$ & Preterm labor \\
\hline \multicolumn{7}{|l|}{ Full-term } \\
\hline FT1 & 3495 & 39 & $\mathrm{~F}$ & $\mathrm{~N}$ & $\mathrm{~N}$ & Repeat CS \\
\hline FT2 & 3420 & 39 & $M$ & $\mathrm{~N}$ & $\mathrm{~N}$ & Repeat CS \\
\hline FT3 & 3475 & 39 & $\mathrm{~F}$ & $\mathrm{~N}$ & $\mathrm{~N}$ & Repeat CS \\
\hline FT4 & 2955 & 40 & $\mathrm{~F}$ & $\mathrm{~N}$ & $\mathrm{~N}$ & Breech \\
\hline FT5 & 3010 & 38 & $M$ & $\mathrm{~N}$ & $\mathrm{~N}$ & Repeat CS \\
\hline
\end{tabular}

ASevere intrauterine growth restriction. CS, cesarean delivery.

and molecular methods, will be necessary to resolve the presence and dynamics of the fetal microbiota during pregnancy.

In this work, we studied the microbiota of human and mouse dyads to understand these relationships, localize bacteria in the fetus, and demonstrate bacterial viability. In preterm and full-term mother-infant dyads in the operating room at the time of cesarean delivery, we sought to understand the nature of the relationships of the maternal vaginal, placental, and fecal microbiota with the infant's oral and meconium microbiota. To allow for a deeper characterization of the nature of these relationships under controlled conditions, we also studied the materno-fetal microbiota in mice. We used a combination of bacterial DNA sequencing, fluorescence in situ hybridization (FISH), and bacterial culture to determine the presence and viability of the fetal microbiota throughout gestation.

\section{Results}

The human perinatal mother-infant microbiota. We studied the microbiota at the time of birth in a cohort of 10 preterm and full-term mother-infant dyads. Only infants born by cesarean delivery were included to enable sampling of the in utero environment and to avoid exposure to the maternal genitourinary microbiota during vaginal delivery. Eight of 10 mothers did not experience labor before delivery, and 9 of 10 had intact membranes at the time of cesarean delivery. The median gestational age at delivery was 26 (range: 24-29) weeks among the 5 preterm infants and 39 (range: 38-40) weeks among the 5 full-term infants (Table 1). All mothers received a single dose of intravenous antibiotics at the time of cesarean delivery. None of the full-term infants received antibiotics, but 4 of the 5 preterm infants were treated with antibiotics empirically after birth. None of the infants had positive blood cultures.

We collected samples from the 10 mother-infant pairs under sterile conditions in the operating room at the time of cesarean delivery. Samples were collected from the endometrial surface (uterus), placenta, and amniotic membrane, as well as the mother's vagina and rectum. The infant's oral cavity was sampled using a sterile swab within the first minutes after birth. The infant's first meconium stool was collected from each infant at a median age of 1 (interquartile range [IQR]: 1-4) day. Plasma, collected by venipuncture from the mother following delivery, was included as a control.

We used 16S rRNA gene sequencing to characterize the composition and diversity of the microbiota at each body site. We used the Divisive Amplicon Denoising Algorithm (DADA2) to infer 16S rRNA gene amplicon sequencing variants (ASVs), which is superior to conventional clustering of operational taxonomic units in discriminating fine-scale sequence variation and resolving sequencing errors (25). A total of 3789 unique ASVs were present among the samples. The median number of reads per sample was 19,666 (IQR: 15,265-43,291). The median number of ASVs observed in each sample site was not significantly different across sites $(P>0.05$ for all pairwise comparisons by Wilcoxon's rank-sum test with Benjamini-Hochberg correction). Alpha diversity, measured by the Shannon Index, was higher in the mother's plasma than the vagina and the infant's oral cavity (4.03 vs. $2.97, P=0.027$; and 4.03 vs. $2.59, P=0.029$, 
A
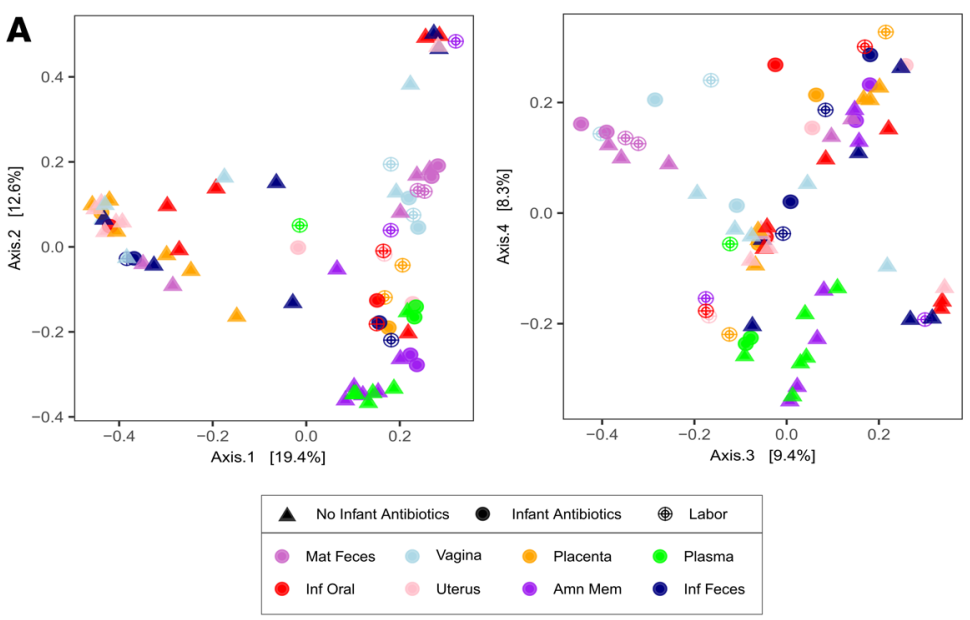

C
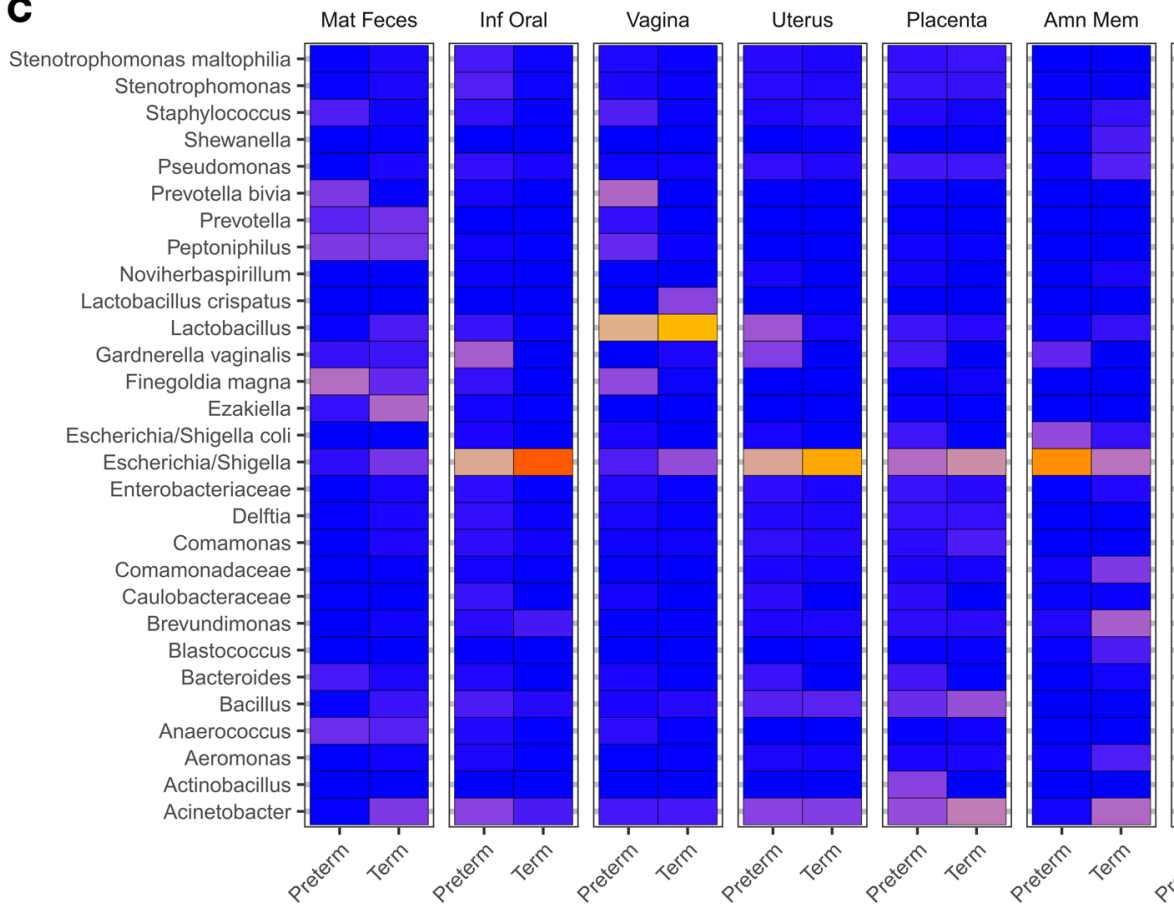

Figure 1. Sequencing-based determination of the microbiota of human preterm and full-term mother-infant dyads delivered by cesarean delivery. (A) Principal coordinates analysis of samples based on Bray-Curtis distances. (B) Pairwise comparisons of Bray-Curtis distances between sample sites by adonis PERMANOVA with Benjamini-Hochberg adjustment. The $R^{2}$ value and $P$ value (in parentheses; highlighted if $P<0.05$ ) are noted for each comparison. (C) Heatmap of the relative proportion of the top bacterial taxa (lowest assigned taxonomy) represented within each sample site in premature and full-term mother-infant pairs.

respectively) but did not differ significantly between the other sites. The placental microbiota of the mother who experienced a rupture of membranes before preterm delivery contained the highest number of observed ASVs (552 vs. range 38-241), but similar Shannon diversity (2.92 vs. 2.18-4.18), compared to the other placental samples. A greater proportion of ASVs was shared between maternal and infant sites within individual mother-infant dyads than between unrelated mothers and infants (Supplemental Table 1; supplemental material available online with this article; https://doi.org/10.1172/jci.insight.127806DS1). A relatively lower proportion of ASVs was shared between the mother's plasma and the infant samples.

We examined the relationship of the microbiota composition between body sites, including all mother-infant pairs. Overall variation between sites was higher than variation within sites $\left(R^{2}=0.191 ; P=0.001\right.$ by the adonis permutational analysis of variance [PERMANOVA], Figure 1A). In pairwise comparisons between sample sites, the microbial communities within the uterus and placenta were distinct from the amniotic membrane microbiota that shared more similarity with the plasma microbiota (Figure 1, A and B). 
A

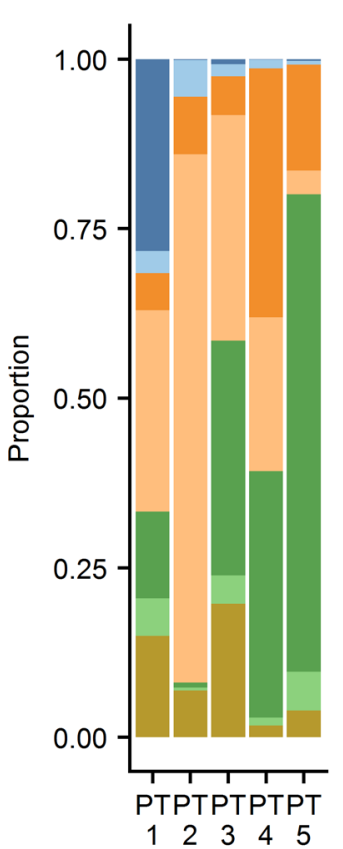

Subject
INF FECES

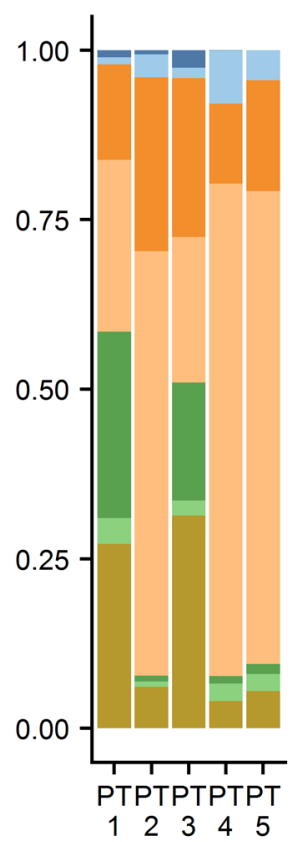

Subject

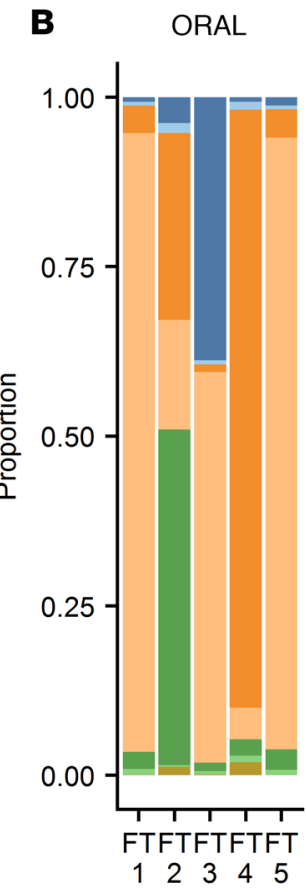

Subject

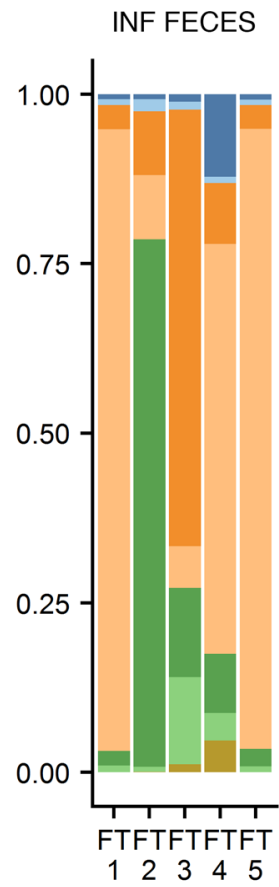

Subject

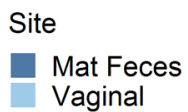

- Vaginal

Uterus

Placenta

Amn Membranes

Plasma

Unknown

Figure 2. Proportions of the infant microbiota attributed to maternal and in utero sources. Predicted sources of the infant microbiota were determined using SourceTracker (26). The proportion of infant microbiota attributed to the maternal and in utero sources are shown for the infant's oral cavity and feces (i.e., meconium) among preterm (A) and full-term (B) mother-infant dyads. The subjects did not experience labor or rupture of membranes before the delivery, with the exceptions of subject PT3 (preterm labor and rupture of membranes) and subject PT5 (preterm labor without rupture of membranes). The preterm infants PT1, PT2, PT3, and PT5 received antibiotics after birth.

The placental and uterine communities were distinct from the maternal fecal microbiota but did not differ significantly from the microbiota of the vagina, infant oral cavity, or infant meconium. The microbial communities of the infant meconium and oral cavity varied between subjects in their relationship to other sites but were distinct from the microbiota of the maternal feces and plasma.

Among many of the preterm and full-term mother-infant dyads, the in utero environment and infant sample sites were enriched for ASVs assigned to the genera Escherichia and Acinetobacter (Figure 1C). Preterm or full-term gestational age accounted for a minor proportion of the variation in microbial community composition (adonis PERMANOVA $R^{2}=0.020 ; P=0.008$, stratified by body site). Many bacterial taxa were shared across multiple maternal and infant sites within individual dyads (Supplemental Figure 1).

We used source tracking of bacterial ASVs in the infant's oral cavity and meconium to determine potential in utero or maternal origins of the infant microbiota (26). We included the uterus, placenta, amniotic membrane, maternal feces, vagina, and plasma as candidate sources of the infant microbiota. The model predicted the placental microbiota as a common origin for the immediate postnatal infant oral and meconium microbiota (Figure 2). In some of the dyads, a higher proportion of the microbiota aligned with the amniotic membrane or uterine sources. For validation of the model, we input individual maternal and in utero sites as "sinks" and evaluated the other sites from the entire cohort as potential sources. The model generally predicted the same site type as the source of microbiota for each sample (Supplemental Figure 2). In total, our results demonstrate the presence of microbial DNA in the meconium and oral cavity at birth in infants born from 24 to 40 weeks of gestation and predict an origin from the in utero environment.

The materno-fetal microbiota in mice. To allow for more in-depth investigation of the in utero microbiota under controlled conditions, we studied the dynamics and affiliations of the murine materno-fetal microbiota. We performed operative deliveries on pregnant dams under sterile conditions at time points ranging from mid- to late gestation. Comprehensive samples from the mother, the fetuses, and the in utero environment were sequenced at the $16 \mathrm{~S}$ rRNA locus to characterize the microbiota at each site. A total of 3050 ASVs were identified, with a median of 16,940 reads per sample. Median diversity within samples, measured by 

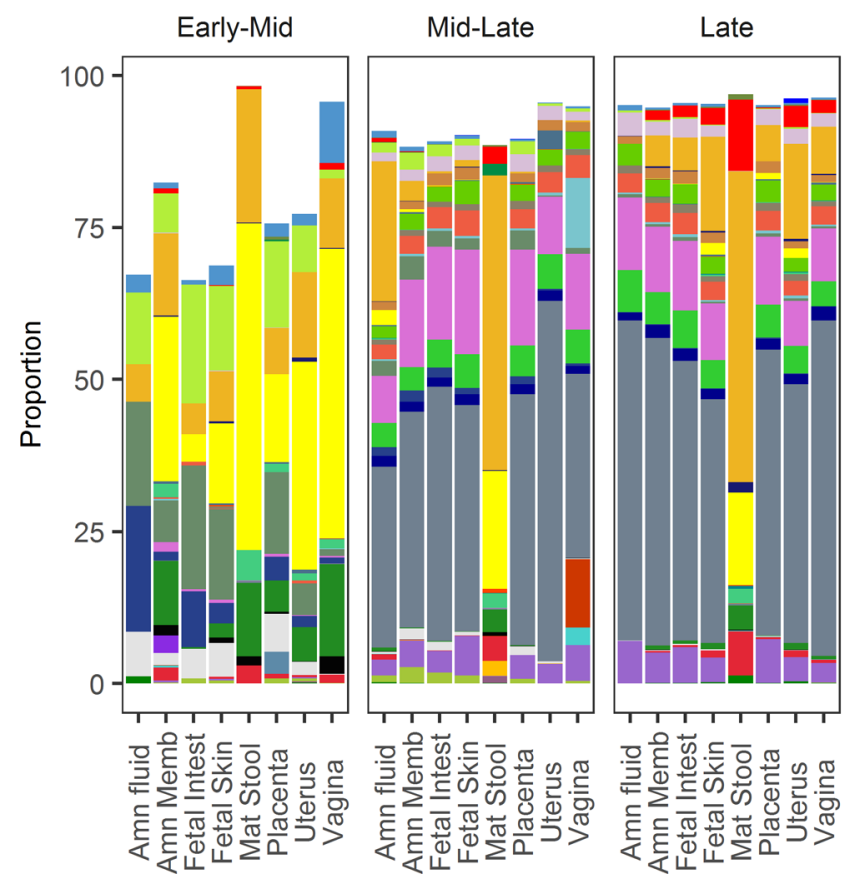

Lowest Assigned Taxonomy

Acidiphilium
Acinetobacter
Akkermansia muciniphila

Allobaculum

Alloprevotella

Anoxybacillus

Bacillus

Bacteroidales_S24-7_group

Bifidobacterium

Brachybacterium

Candidatus_Arthromitus

Chloroplast

Clostridium_sensu_stricto_1

Comamonas

Coriobacteriaceae_UCG-002

Desulfovibrio

Domibacillus

Enterobacteriaceae

Enterococcus
env.OPS_17

Escherichia/Shigella

Exiguobacterium

Hydrogenophilus islandicus

Kurthia

Kurthia gibsonii

Lactobacillus

Lactobacillus intestinalis

Lactococcus

Meiothermus silvanus

Mucispirillum schaedleri

Olsenella

Pantoea

Parasutterella

Romboutsia

Sphingobacterium

Tepidimonas ignava

Thalassospira

Turicibacter

Figure 3. Relative abundance of top bacterial taxa in murine maternal and fetal microbiota across gestational stages. The top 15 bacterial taxa (lowest assigned taxonomy) within each maternal or fetal site is shown among early-mid (E14-16), mid-late (E17-18), or late (E19-20) gestation mice. Samples from 2 dams and 3-5 fetuses per dam are represented.

the Shannon Index, was highest in the maternal feces and lowest in the vagina (3.01 and 1.94, respectively), but the sites did not differ significantly from each other following adjustment for multiple comparisons.

In the next-generation sequencing-defined composition of the microbiota in the maternal and fetal tissues, many shared bacterial taxa were found in the in utero and fetal compartments (Figure 3). In mice sampled in early-mid gestation (E14-16, $n=2$ dams and $\geq 3$ fetuses/dam), the microbiota of the fetal compartments contained many of the same ASVs as the maternal fecal microbiota, including ASVs within the genera Candidatus Arthromitus, Bacteroidales S24-7, Lactobacillus, and Desulfovibrio. In mice sampled in mid-late (E17-18) or late (E19-20) gestation ( $n=2$ dams and $\geq 3$ fetuses/dam/time point), the in utero and fetal compartments shared greater overlap with ASVs identified in the placental and vaginal microbiota, including Kurthia gibsonii and Escherichia/Shigella ASVs. The taxon Bacteroidales S24-7 dominated the maternal fecal microbiota and was concurrently represented in the in utero and fetal samples at these later time points. As controls, we included 2 samples from the sterile instruments used to collect samples from the fetal sites ("control pick") in the sequencing run. We also collected blood from the animals immediately before the operative deliveries. The most abundant ASVs in the fetal intestine were often represented in the blood at lower abundances but not in the negative control samples (Supplemental Figure 3). For further confirmation of the fetal gut microbiota, we performed FISH on fetal intestinal tissue sections using the eubacterial probe EUB338. The specificity of the probe was also tested using sections treated with no probe or a sequence-scrambled probe. The EUB338 probe was visualized at low abundance throughout the fetal intestine (Figure 4). The no-probe and scrambled-probe controls did not show the staining seen in the EUB338-probed sections (representative images are shown in Figure 4).

Similar to the human data, microbiota community composition based on Bray-Curtis distances varied significantly between body sites in a PERMANOVA model $\left(R^{2}=0.119 ; P=0.003\right.$, Supplemental Figure $4 \mathrm{~A})$. In pairwise comparisons, the maternal fecal samples differed significantly from all other sites $\left(R^{2}\right.$ values of 0.168 to 0.302 ). The variation between other sites was not significantly greater than within sites for the remaining pairwise site comparisons. The community composition shifted along PC1 between samples collected in early-mid and late gestation (median -0.38 vs. $0.41 ; P<0.001$, Supplemental Figure 4B).

Using bacterial source tracking of the $16 \mathrm{~S}$ rRNA gene ASVs to determine the most likely maternal microbiota sources for the fetal gut and skin, the predicted source of the fetal microbiota varied by gestational age at the 

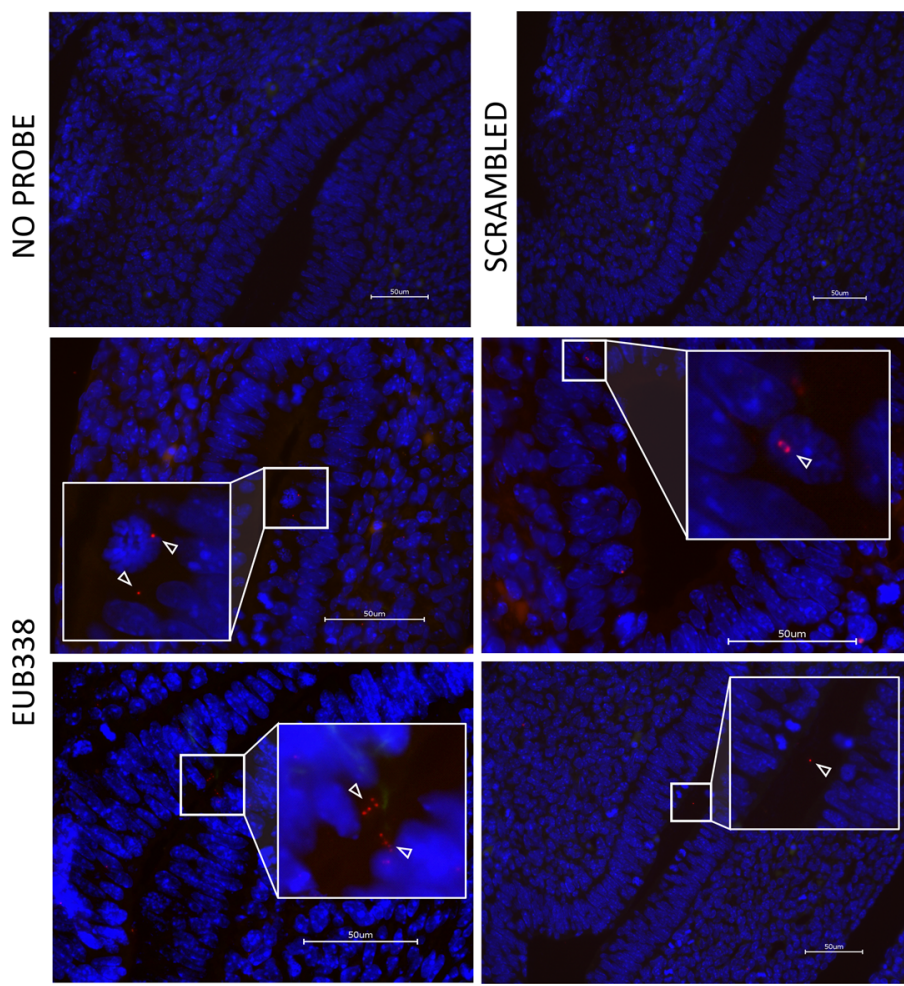

Figure 4. Identification of bacterial DNA in the murine fetal intestine by FISH of the 165 rRNA gene. Intestinal tissue sections were obtained from fetal mice at E12-14 and were stained with DAPI and probed with Cy3-labeled eubacterial probe EUB338 and a scrambled-probe control. Images were acquired by confocal microscopy. Scale bar: 50 $\mu \mathrm{m}$. Magnification, $10 \times$

time of sampling (Figure 5). In mid-late (E17-18) and late (E19-20) pregnancies, the microbiota of the fetal gut and skin were more commonly attributed to the placenta, followed by the amniotic membrane. The predicted source of the fetal microbiota in early-mid gestation (E14-16) was more variable than measured in the later-stage samples. Compared with the later stages, the fetal bacterial ASVs had an increased predicted relationship to the control pick samples in some of the samples, potentially reflecting a reduced bioburden and increased risk for sampling bias. Related to this same issue, the model produced more low-confidence sources grouped as "unknown." Despite these challenges, unique ASVs distinguished fetal samples from controls, suggesting early fetal exposure to bacteria. Together, the results suggest that the fetal microbiota is dynamic during gestation.

We next sought to determine whether viable and cultivatable bacteria were present in the murine in utero compartment and fetus. Samples collected from pregnant dams and their fetuses were homogenized, plated on culture media, and incubated under anaerobic and/or aerobic conditions with media- and instrument-alone controls to exclude environmental contamination during the sample collection. Viable bacteria were detected in the fetal and in utero sites (Figure 6). In some cases, strains with high sequence identity were isolated from more than 1 site across individual fetal and in utero sites. Positive cultures were more common in mid-gestation, whereas the samples obtained near full term did not yield positive cultures despite the presence of bacterial DNA sequences. Lactobacillus strains were the most common isolates in the fetal gut and in utero samples. Lactobacillus ASVs were also identified in the sequencing data, though they accounted for a minor portion of the microbiota (range: $0 \%-30.6 \%$ of the total gut microbiota among individual fetuses). Other bacterial isolates from the fetal and in utero samples included strains of Escherichia, Enterococcus, Bacteroides, Bacillus, and others (Figure 6). Many of these taxa were also cultured from maternal fecal and vaginal samples, and occasionally colonies with high-confidence sequence identities were isolated concurrently from fetal and maternal sites. For example, Enterococcus strains isolated from the placenta were similar to strains in the maternal vagina and feces. We collected samples from the maternal skin and blood for comparison. Cultured organisms from the skin were typically distinct from the in utero isolates and included Streptococcus, Rothia, Bacillus, Staphylococcus, Streptomyces, and Brevibacillus spp. A single colony (Streptococcus sp.) was detected in blood from 1 of the dams; all other blood cultures were negative. To exclude contamination from maternal 

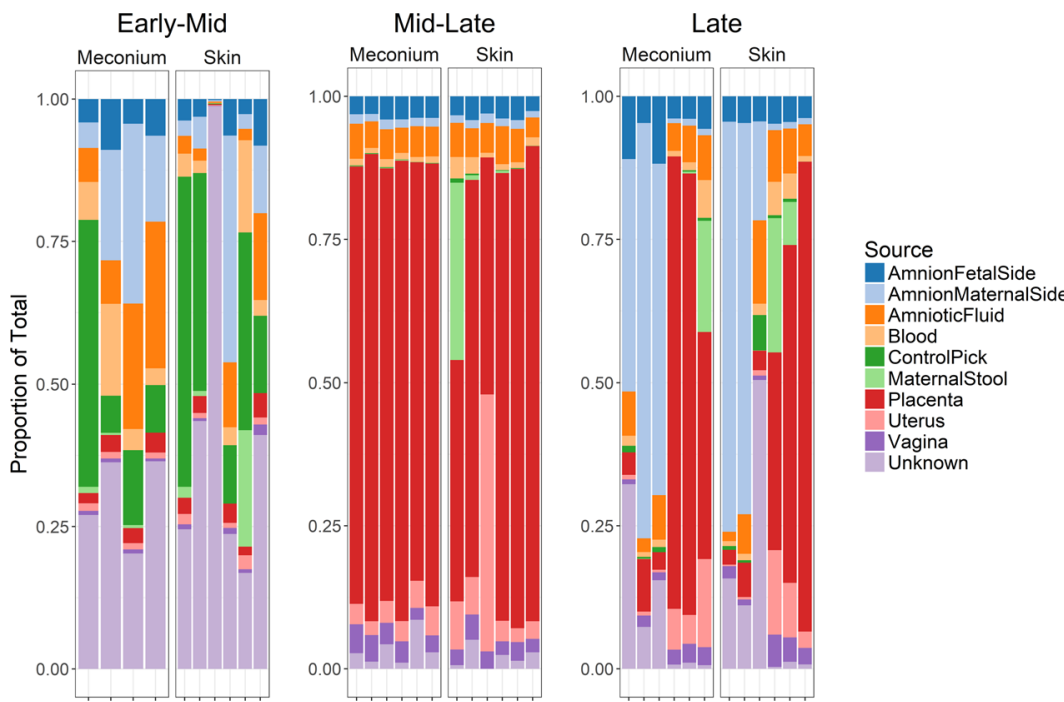

Figure 5. Predicted sources of the murine fetal microbiota. Bacterial source tracking was used to determine the likely sources of bacterial DNA in the gut and skin of early-mid (E14-16), mid-late (E17-18), and late (E19-20) gestation fetuses. Samples from 2 dams and their corresponding fetuses are represented.

skin flora, the skin of pregnant dams $(n=2)$ was topically coated with a spectinomycin-resistant $E$. coli before dissection. The skin was then cleaned using the same procedures as the previous experiments. Maternal and fetal tissues were plated on MacConkey agar with spectinomycin. Maternal skin swabs collected following application of the E. coli solution showed growth of the antibiotic-resistant strain, while all other maternal and fetal tissues were negative, demonstrating a lack of contamination from the maternal skin flora during dissection (data not shown).

We next sought to determine whether bacteria could be cultivated from the fetal environment following maternal colonization during pregnancy. Pregnant dams were treated with streptomycin by oral gavage once daily for 2 consecutive days in early gestation to make the gut microbiota permissive to colonization by an E. coli administered by gavage (E5-6; Figure 7A). The following day (E7), the dams were treated by oral gavage with a streptomycin-resistant uropathogenic E. coli strain (UTI89/pCOMgfp [UTI89]; $1 \times$ $10^{8}$ colony-forming units [CFU]) (27). The dams appeared well, appeared identical to untreated controls, demonstrated no adverse effects, and had no spontaneous pregnancy losses following treatment. Tissues were collected from the dams and their fetuses in early (E9) or late (E18) gestation and plated on Luria agar containing streptomycin. In early gestation (E9), streptomycin-resistant $E$. coli was cultured from the maternal cecum, blood, and uterus and the fetus (Figure 7B). Bacterial counts ranged from 10 to $10^{3} \mathrm{CFU}$ per fetus (excluding placenta). Few or no E. coli colonies were cultured from the vagina. In late gestation (E18) following prior administration of E. coli on E7, the administered bacteria persisted in the maternal cecum but were no longer detected in the in utero environment or fetus, with the exception of 1 fetus with low counts in the amniotic fluid (Figure 7C). Control cultures performed in the mouse colony and separately housed littermates demonstrated that the animals were not inherently colonized with streptomycin-resistant bacteria (data not shown). Together, these results demonstrate that viable $E$. coli can be cultured from the fetal environment in early gestation following colonization of the murine maternal gut.

To ensure that bacteria identified in cultures and FISH from murine fetuses were not due to cross contamination from adjacent maternal sites, the following control experiment was conducted. Pregnant dams at E14 were anesthetized and placed in a sterile surgical field. The uterus was surgically exposed, and the external surface was coated with a kanamycin-resistant $E$. coli. Every other fetus within the uterus was then microinjected through the uterine wall into the fetal liver with a spectinomycin-resistant $E$. coli using a hand-pulled glass pipette. After placing tight ligatures to separate each fetal-placental unit, the uterine wall was harvested, followed by the amniotic sac, followed by each individual fetus. The maternal and fetal tissues were homogenized in sterile fluid and plated on antibiotic-containing agar plates, and the antibiotic-resistant $E$. coli were enumerated. As shown in Supplemental Figure 5, cultures from the uninjected fetuses and accompanying amniotic sacs did not grow either bacterial strains. Cultures of fetuses injected with 


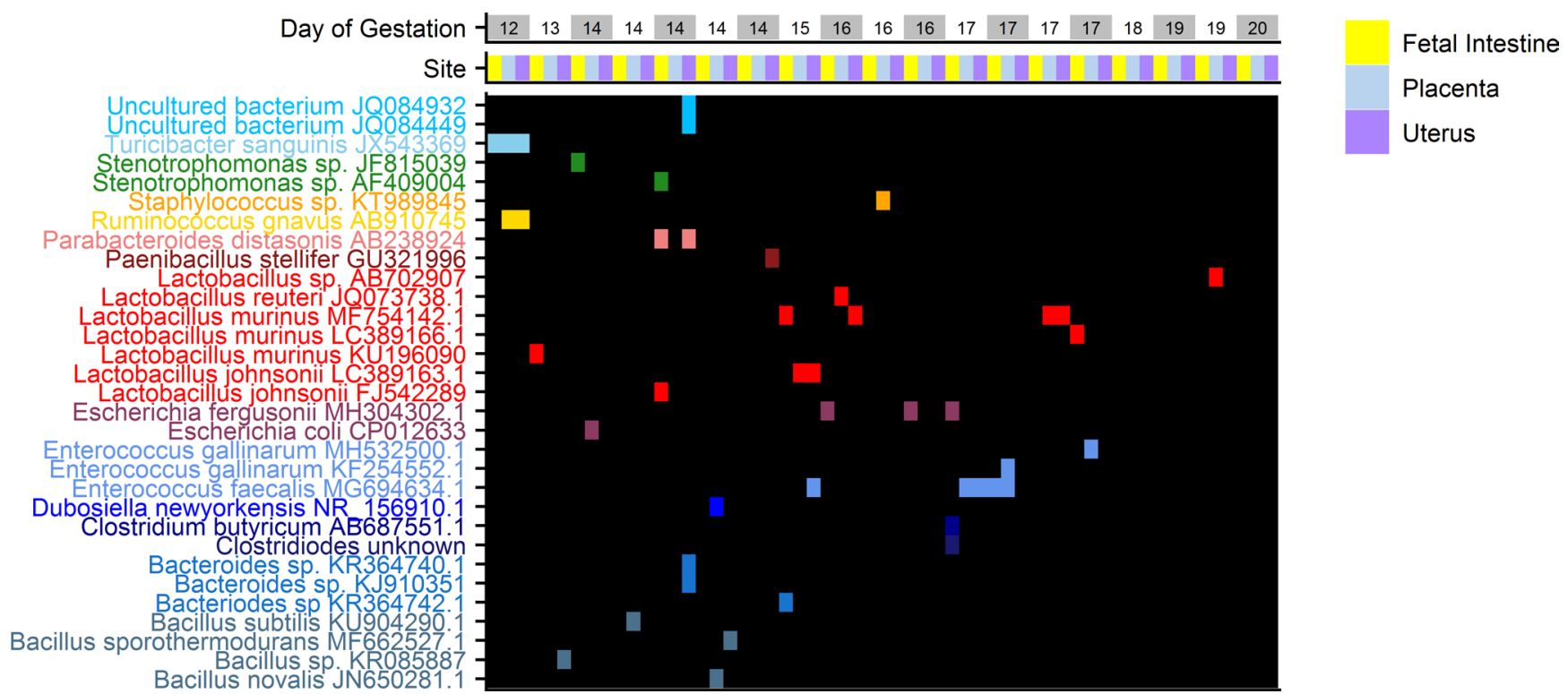

Figure 6. Bacterial strains cultured from the murine fetal intestine, placenta, and uterus. The top gray-and-white bar indicates individual dams and the embryonic day at the time of sampling, followed by the anatomic site from which the bacterial strains were isolated. Colored squares in the heatmap represent the sites with positive cultures. Fewer samples had positive cultures in late gestation. Strains were identified by Sanger sequencing of the $16 \mathrm{~S}$ rRNA gene (8F/1492R). The accession number for the top match identified by the Basic Local Alignment Search Tool (BLAST) is shown for each isolate (isolates without a match of at least $94 \%$ sequence identity were designated as unknown).

the spectinomycin-resistant strain of $E$. coli and the accompanying amniotic sacs grew only the injected strain and not the kanamycin-resistant strain used to intentionally contaminate the uterus. Uterine samples demonstrated variable growth of both $E$. coli strains indicating some potential contamination from the fetal harvest to the uterus. However, these controls suggest that fetal cultures do not reflect contamination from maternal sources, such as the uterus.

\section{Discussion}

Our study expands the limited body of literature that challenges the notion that the fetus develops in a sterile environment $(7-11,14,18,24,28)$. Strengths of this work include complementary human and mouse sampling, visualization of a fetal microbiota in mice, demonstration of gestational stage dynamic changes in maternal and fetal sites, and viability among cultivatable constituents. We observed the presence of an in utero microbiota in human mother-infant dyads undergoing cesarean delivery and in mice sampled under sterile conditions in the laboratory. Our observations of the presence of bacterial DNA in the fetus and in utero environment were corroborated by visualization of bacteria in the fetal gut by FISH and culture data showing the presence of viable bacteria in the murine fetal gut.

Although our data from humans and mice indicate that the fetus is exposed to bacterial DNA throughout mid-gestation and late gestation, we observed a temporal shift in the composition and predicted source of the microbiota as pregnancy progressed. Although differences between the microbiota in mid-gestation and late gestation appeared less marked in the human mother-infant dyads than mice, there was substantial interindividual variation, and studies in larger cohorts would be necessary to make definitive conclusions. Our culture data suggest that the murine fetus is exposed to viable and cultivatable bacteria in mid-gestation that are subsequently noncultivatable in late gestation despite sequencing demonstrating the presence of organisms in this time frame. We speculate that changes in immune regulation at the materno-fetal barrier during gestation may lead to differences in the ability of microorganisms to access and persist in the fetal environment. It is also possible that some microbes may persist in a viable but noncultivatable state (29). The mouse model we describe may be useful to elucidate the nature of the interactions between the maternal microbiota and the developing fetus in future work.

The presence of a fetal microbiota remains an area of intense interest and debate (30). The low bacterial biomass of the intrauterine environment makes it difficult to discern from environmental contamination that can occur during sample collection and preparation $(16,20)$. A recent study in a large cohort of women found 
A
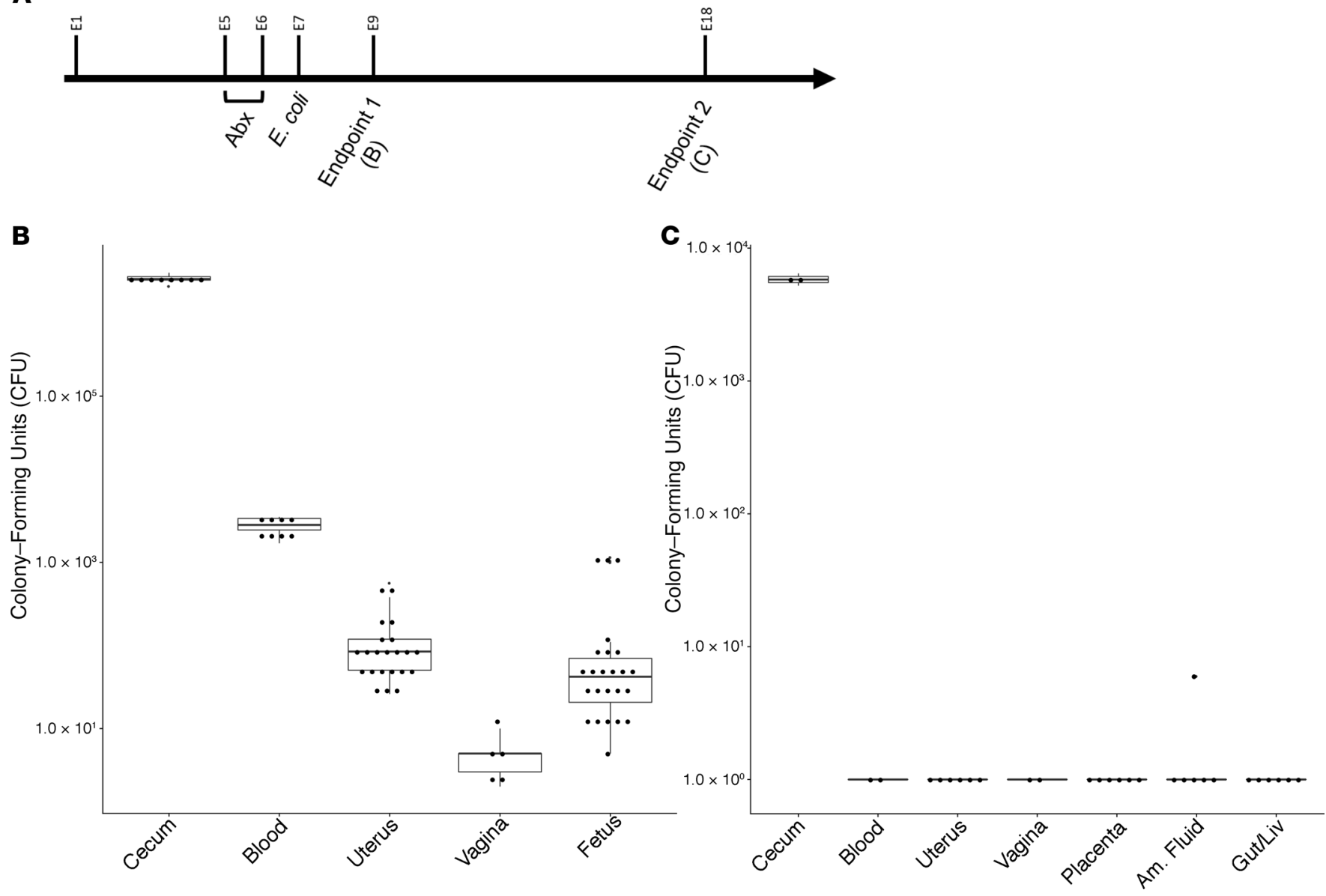

Figure 7. Cultivation of streptomycin-resistant $\boldsymbol{E}$. coli from maternal and fetal tissues following maternal colonization. (A) Timeline for treatment of dams with antibiotics (Abx; streptomycin), colonization with streptomycin-resistant $E$. coli by oral gavage, and collection of maternal and fetal tissues. (B) Colony-forming units (CFU) counts per plate (100 $\mu$ homogenate/plate) from the maternal and fetal tissues in early gestation (E9). (C) CFU counts per plate (100 $\mu$ l homogenate/plate) from the maternal and fetal tissues in mid-late gestation (E18). The box plots depict the minimum and maximum values (whiskers), the upper and lower quartiles, and the median. The length of the box represents the interquartile range.

that most of the bacterial DNA sequences identified in the terminal villi of the human placenta could be attributed to environmental contamination (22). However, human-associated bacterial 16S rDNA signatures were identified in the placentas of women who underwent cesarean delivery without labor that could not be attributed to laboratory contamination. These taxa included Lactobacillus signatures and were described as "delivery-associated signals" given their presence in higher frequency in the placentas of women with vaginal delivery or labor before delivery. Our control culture experiments in mice show that Lactobacillus and other microbes were present in the fetal tissues and were unlikely to be acquired by contamination from adjacent tissues. Our sequencing and cultivation results identified shared microbial signatures within individual mother-infant dyads and distinctions between the fetal/intrauterine samples and controls. Source tracking suggested the placenta may be an important source of the microbiota in both humans and mice.

Although many of the samples did not yield viable organisms in culture, a variety of organisms were cultivated from the murine fetal gut, placenta, and uterus, including Lactobacillus, as well as Enterococcus, Escherichia, and others. These organisms are common constituents of the human neonatal microbiome, and bacterial DNA from these taxa has been identified in meconium, amniotic fluid, and the placenta in previous studies (7-9, 13, 31, 32).

Despite a small sample size, we noted that the samples collected earliest in gestation (E12-16) were more likely to yield positive cultures than samples collected near full term, which were typically sterile. Another recent study reported sterile cultures from the murine fetal gut lumen at a single time point in mid-late gestation (E17), despite the presence of bacterial DNA (18). Of the cultured isolates in our study, 
invasion of the placenta by Escherichia and other organisms often invokes a systemic inflammatory response in the fetus, whereas the presence of Lactobacillus in the placenta has been associated with a suppressive effect (33). We found that the fetal tissues of dams intentionally colonized with antibiotic resistance-marked E. coli in pregnancy contained cultivatable E. coli in early but not late gestation. Although the dams did not exhibit overt clinical infection, E. coli was cultivated from the blood and cecum. These results suggest that the bacteria reached the fetus via the gut or bloodstream, but further studies are needed to delineate the source and route of passage to the fetal environment. We did not perform bacterial culture on the human samples collected in our study. Collado et al. report the presence of viable organisms, including Propionibacterium and Staphylococcus, from the placenta and amniotic fluid at the time of cesarean delivery in a cohort of full-term mothers and infants (8). If live bacteria are indeed present in the human fetal compartment, the mechanisms by which they bypass physical and immune barriers at the materno-fetal interface and the factors that prevent progression to clinical infection remain to be determined.

Successful pregnancy requires coordination and balance between maternal and developing fetal immunity. Microbes have important roles in immune homeostasis and dysregulation. The presence of fetal organisms or their molecular signatures may prime fetal mucosal immunity and ready the tissues for colonization following birth $(17,32)$. We observed microbial DNA signatures, cultivability, and FISH evidence of bacteria during a critical window of fetal immune development. Innate lymphoid cells are reported in the developing murine fetal liver, intestine, lung, and spleen starting at E12.5-14.5, with lymph nodes developing by E16.5, during which time frame we observe direct evidence of microbial colonization of the fetus (34). Human fetal intestinal Innate Lymphoid Cells (ILCs) produce key cytokines, including IL-13, IL-22, and IL-17A, which in turn contribute to immune and tissue development (35-37). A recent study demonstrated the presence of tissue-resident memory-like $\mathrm{T}$ cells in the human fetal intestine that secrete proinflammatory cytokines in higher levels upon stimulation than naive T cells (38). These results suggest that the fetal intestine is exposed to foreign antigens, but it is not known whether these antigens are microbial. Future work will distinguish the role of direct fetal exposure to microbes from other mechanisms of fetal immune stimulation, such as exposure to microbial ligands originating from the maternal gut.

In summary, we describe the presence of bacterial DNA and viable bacteria in the in utero environment. Although complex mechanisms have evolved to protect the developing mammalian fetus from exposure to pathogens and toxins, the discovery of bacterial signatures in the in utero environment in humans and mice suggests the coexistence of refined mechanisms for controlled fetal exposure to the maternal microbiota. Even transient exposure to bacterial antigens in the fetal environment may provide important evolutionary advantages for early immune maturation. The potential implications of these findings on fetal development highlight the need for further work to fully elucidate the mechanisms by which the fetus comes into contact with the maternal microbiota.

\section{Methods}

Study population. We prospectively enrolled premature (birth gestational age $<30$ weeks and birth weight $<$ $1000 \mathrm{~g}$ ) and full-term (birth gestational age $>37$ weeks) mother-infant dyads. Exclusion criteria were vaginal delivery, known congenital anomalies, clinical chorioamnionitis, and maternal autoimmune disease, diabetes, or immunodeficiency. All mothers received routine surgical antibiotic prophylaxis at the time of cesarean delivery, but mothers who had received any additional antibiotics within 48 hours preceding delivery were excluded.

Human sample collection. Samples were collected by trained research personnel and obstetricians in the operating room under sterile conditions at the time of cesarean delivery. Vaginal and rectal samples were collected by inserting a sterile swab into the distal vagina or rectum. Uterine samples were obtained by swab from the endometrial lining of the anterior uterine wall. After delivery, the placenta was placed in a sterile basin, and the surface was swabbed around the cord insertion site. A $1 \mathrm{~cm} \times$ $1 \mathrm{~cm}$ section of the amniotic membrane was excised adjacent to the site of membrane rupture. The infant's oral cavity was swabbed in the operating room immediately after delivery. Premature infants were admitted to the neonatal intensive care unit; full-term infants were admitted to the newborn nursery and roomed in with their mothers. The infant's first meconium stool was collected from the diaper, immediately transferred to a sterile vial, and frozen.

Mouse sample collection. Pregnant C57BL/6 mice (Charles River Laboratories and the Jackson Laboratory) underwent operative delivery under sterile conditions in the laboratory. The mice were housed 
in a pathogen-free barrier facility before delivery. Deliveries were timed throughout mid to late gestation, from gestational day 12 through 20, with pregnancy dating based on vaginal plug dates. The pregnant mice were deeply anesthetized with isoflurane immediately before delivery. The mice were shaved and scrubbed with betadine and ethanol. Blood was collected by intracardiac puncture. A vaginal sample was collected using a sterile plastic brush. The animals were placed under sterile drapes and a midline abdominal incision was made, exposing the gravid uterus. Individual fetal-placental units were separated from the surrounding units by suture ligation to allow for dissection of individual units. The external uterine surface was sterilized with 70\% ethanol. Amniotic fluid was aspirated from 2 ligated fetal units per mother. The remaining units were dissected for the collection of tissue specimens. First, the uterine wall was opened and a portion resected and retained. Next, the amniotic membrane was carefully peeled off the fetus using a sterile plastic pick. The whole placenta was collected en bloc. The skin of each fetus was swabbed and the fetal intestines were collected. After all tissues had been collected, the maternal colon was opened and a fecal sample was obtained. The plastic brush-tipped picks used to collect mouse vaginal, amniotic membrane, and fetal skin samples were included in the sequencing run as controls.

In a subset of the mice, 1-2 whole fetal units were removed and fixed in 10\% neutral buffered formalin (NBF) for up to 5 days, followed by 3 washes in phosphate-buffered saline (PBS) and 48-72 hours of incubation in $30 \%$ sucrose at $4^{\circ} \mathrm{C}$. The fetuses were then snap-frozen in OCT medium and placed at $-80^{\circ} \mathrm{C}$ for cryosectioning.

$16 S$ rRNA gene sequencing and FISH. After collection, samples were stored at $-80^{\circ} \mathrm{C}$ until further processing. Genomic DNA was extracted using bead-beating and commercial extraction kits (Zymo Research). Paired-end, barcode-indexed primers (Integrated DNA Technologies; 515F 5'-GTGCCAGCMGCCGCGGTAA-3'; 806R 5'-GGACTACHVGGGTWTCTAAT-3') were used to amplify the $515 \mathrm{~F} / 806 \mathrm{R}$ region of the $16 \mathrm{~S}$ rRNA gene. For mouse samples, PCR reagents were pretreated with shrimp DNAse (Thermo Fisher Scientific) to remove contaminating double-stranded DNA. The DNAse was inactivated by heating at $37^{\circ} \mathrm{C}$ for 30 minutes and $65^{\circ} \mathrm{C}$ for 10 minutes before adding the genomic DNA template. The PCR was run according to the Earth Microbiome Project protocol (39). Negative controls (no DNA template) were run for each extraction kit and PCR. Amplicons were pooled in equimolar concentrations and purified by gel extraction (GeneJET PCR Purification Kit, Thermo Fisher Scientific). Amplicons were sequenced on the Illumina MiSeq platform. Sequencing data were deposited in the National Center for Biotechnology Information's Bioproject number PRJNA557826. The resulting amplicon sequences were trimmed and chimera reduced, and paired ends were merged using DADA2 (25).

FISH detection of bacteria in fetal tissues. We performed FISH on fetal tissues using the universal 16S rRNA gene probe EUB338 labeled with Cy3 (IDT DNA Technologies). Fetal tissue sections were fixed in $10 \%$ NBF for 10 minutes, washed in PBS, fixed in Carnoy's solution for 5 minutes, and washed again. The tissue was permeabilized with $0.2 \mathrm{M} \mathrm{HCl}$ for 20 minutes. Slides were then incubated with hybridization buffer (100 mM Tris- $\mathrm{HCl}, 0.9 \mathrm{M} \mathrm{NaCl}, 0.1 \% \mathrm{SDS}$, and $35 \%$ formamide, $\mathrm{pH} 7.5$ ) at $46^{\circ} \mathrm{C}$ for 30 minutes before adding the probe at a final concentration of $5 \mathrm{ng} / \mu \mathrm{l}$. Following overnight incubation at $46^{\circ} \mathrm{C}$, slides were washed in PBS, rinsed with water, and then stained with DAPI. Slides were examined using the Leica DM6000CS SP5 upright confocal microscope with original image magnification at $\times 40 / 1.25-0.75$ oil. Images were analyzed with Leica LAS AF 2.3 software. Composite images were obtained by combining 3 color channels: red (probe), blue (DAPI), and green (autofluorescence). The fetal gut sections were also imaged with no probe and with a scrambled probe (5'-/Cy3/ACTCCTACGGGAGGCAGC-3'; IDT DNA Technologies) as controls.

Bacterial culture. In a separate group of pregnant mice, samples were collected and immediately homogenized in sterile PBS solution ( $250 \mu \mathrm{l}$ for each tissue specimen and $500 \mu 1$ for fecal specimens). Samples collected by swab or brush were agitated using a vortex mixer (Thermo Fisher Scientific) for 60 seconds. Fifty-microliter aliquots of each homogenized or vortexed sample were plated on various media, including blood-heart infusion agar with vitamin $\mathrm{K}$ and hemin; chocolate agar; deMan, Rogosa, Sharpe agar (MRS) agar; and MacConkey agar (Difco). Negative control plates without tissue specimens were prepared in the same manner to exclude contamination of the laboratory instruments and environment. Plates were incubated in aerobic (with $5 \% \mathrm{CO}_{2}$ ) and anaerobic conditions $\left(0.2 \% \mathrm{O}_{2}, 9.9 \% \mathrm{CO}_{2}, 9.9 \% \mathrm{H}_{2}, 80 \% \mathrm{~N}_{2}\right.$; Anoxomat, Mart Microbiology BV) at $37^{\circ} \mathrm{C}$. Plates were examined daily for growth and incubated for up to 72 hours. 
Single colonies representing each unique morphology on the plate were streaked onto the same agar media type and incubated in the same conditions. After regrowth of isolates, single colonies were selected for PCR amplification of the $8 \mathrm{~F} / 1492 \mathrm{R}$ region of the $16 \mathrm{~S}$ rRNA gene. Amplicons underwent Sanger sequencing, and taxonomy assignments were made using the BLAST (40).

Intestinal colonization of specific pathogen-free pregnant mice by E. coli. Pregnant dams were treated with streptomycin $20 \mu \mathrm{g}$ in $100 \mu \mathrm{l}$ of PBS daily for 2 consecutive days (E5-6), followed by treatment the following day (E7) with a streptomycin-resistant derivative of E. coli strain UTI89. Blood and tissues were collected from the dams and their fetuses at E9 or E18, diluted in sterile PBS, homogenized, and plated (100 $\mu 1$ homogenate per plate) on Luria agar (Genesee Scientific) supplemented with $100 \mu \mathrm{g} / \mathrm{ml}$ of streptomycin to select for the resistant $E$. coli strain. Given the small size and delicate tissues of the early gestation fetuses, whole fetuses were collected en bloc and homogenized in early gestation (E9). In late gestation fetuses (E18), gut and liver were dissected away from the rest of each fetus and homogenized. Cultures were incubated for 24 hours at $37^{\circ} \mathrm{C}$. Colony counts were recorded for each plate.

Control experiments for E. coli materno-fetal tissue cross contamination. Timed matings of SPF C57BL/6 mice (The Jackson Laboratory) were established, and female mice were monitored for the presence of a vaginal plug, which was designated E1. On E14 the pregnant mice were anesthetized with isoflurane and placed onto sterile drapes. A midline incision was made to expose the uterus. The exterior of the uterus was swabbed with a solution of $1 \times 10^{5} \mathrm{CFU} / \mathrm{ml}$ of a kanamycin-resistant E. coli (UTI89). Following this, alternating fetuses were injected using a hand-pulled glass pipette with $5 \mu \mathrm{l}$ of a $1 \times 10^{5} \mathrm{CFU} / \mathrm{ml}$ solution of a spectinomycin-resistant $E$. coli $(\Delta 6 \mathrm{H})$ into the fetal liver. Each fetal-placental unit was then ligated with a sterile surgical suture to allow for individual dissection. First, the uterine wall was carefully removed and placed into sterile PBS. Next, the amniotic sac was peeled off the fetus and placed into sterile PBS. Finally, the entire fetus was placed into sterile PBS. For controls, dissection scissors and forceps were each swirled in sterile PBS at the end of the tissue harvest. A fresh sterile swab and a swab of UTI89 were washed in sterile PBS. Tissue samples were homogenized, and $100 \mu 1$ of the homogenate and controls was plated onto Luria agar with $50 \mu \mathrm{g} / \mathrm{ml}$ kanamycin and LB agar with $50 \mu \mathrm{g} / \mathrm{ml}$ spectinomycin (Thermo Fisher Scientific). The plates were incubated at $37^{\circ} \mathrm{C}$ overnight, and CFU were counted.

Statistics. Alpha diversity was calculated using the phyloseq package (version 1.14.0) in R (41) and described by median and IQR. Comparisons between sites were made using Wilcoxon's rank-sum tests. Two-sided $P$ values of less than 0.05 following Benjamini-Hochberg adjustment for multiple testing were considered statistically significant. Sequence counts were normalized using cumulative sums scaling in the metagenomeSeq package in $\mathrm{R}$ (42). Bray-Curtis distances were compared between samples and sites using adonis PERMANOVA $(43,44)$. SourceTracker was used to investigate the role of each maternal site (e.g., placenta, uterus, vagina, stool) as a potential contributing source of bacteria in the infant's microbiota (26).

Study approval. The human study was approved by the Duke Institutional Review Board. Written informed consent was obtained from the pregnant mothers before delivery. All animal procedures were approved by the Duke and Northwestern University Institutional Animal Care and Use Committees.

\section{Author contributions}

NY, JRM, AM, DB, and PCS contributed to the study conceptualization and design. NY, FAP, JB, JRM, $\mathrm{CP}, \mathrm{SA}$, and PCS performed the experiments and analyzed the data. All authors contributed to writing or critical revision of the manuscript for intellectual content.

\section{Acknowledgments}

We would like to thank the Duke Neonatal-Perinatal Research Unit and the families who participated in the study. The study was supported by The Derfner Foundation (to NY) and the Hartwell Foundation (to PCS). The authors also received support from the NIH (K12 HD043494 and K23 DK120960 to NY; R01GM108494 to PCS and DB), the Zeist Foundation, and the Jean and George Brumley Jr. Neonatal Perinatal Research Institute.

Address correspondence to: Patrick Seed, 225 E. Chicago Ave., Chicago, Illinois 60611-2605, USA. Phone: 312.503.6982; Email: patrick.seed@northwestern.edu. 
1. Olszak T, et al. Microbial exposure during early life has persistent effects on natural killer T cell function. Science. 2012;336(6080):489-493.

2. Gensollen T, Iyer SS, Kasper DL, Blumberg RS. How colonization by microbiota in early life shapes the immune system. Science. 2016;352(6285):539-544.

3. Cox LM, et al. Altering the intestinal microbiota during a critical developmental window has lasting metabolic consequences. Cell. 2014;158(4):705-721.

4. Dominguez-Bello MG, et al. Delivery mode shapes the acquisition and structure of the initial microbiota across multiple body habitats in newborns. Proc Natl Acad Sci U S A. 2010;107(26):11971-11975.

5. Azad MB, et al. Gut microbiota of healthy Canadian infants: profiles by mode of delivery and infant diet at 4 months. $C M A J$. 2013;185(5):385-394.

6. Martín V, et al. Sharing of bacterial strains between breast milk and infant feces. J Hum Lact. 2012;28(1):36-44.

7. Aagaard K, Ma J, Antony KM, Ganu R, Petrosino J, Versalovic J. The placenta harbors a unique microbiome. Sci Transl Med. 2014;6(237):237ra65.

8. Collado MC, Rautava S, Aakko J, Isolauri E, Salminen S. Human gut colonisation may be initiated in utero by distinct microbial communities in the placenta and amniotic fluid. Sci Rep. 2016;6:23129.

9. Ardissone AN, et al. Meconium microbiome analysis identifies bacteria correlated with premature birth. PLoS One. 2014;9(3):e90784.

10. Moles L, et al. Bacterial diversity in meconium of preterm neonates and evolution of their fecal microbiota during the first month of life. PLoS One. 2013;8(6):e66986.

11. Satokari R, Grönroos T, Laitinen K, Salminen S, Isolauri E. Bifidobacterium and Lactobacillus DNA in the human placenta. Lett Appl Microbiol. 2009;48(1):8-12.

12. Bearfield C, Davenport ES, Sivapathasundaram V, Allaker RP. Possible association between amniotic fluid micro-organism infection and microflora in the mouth. BJOG. 2002;109(5):527-533.

13. Rautava S, Collado MC, Salminen S, Isolauri E. Probiotics modulate host-microbe interaction in the placenta and fetal gut: a randomized, double-blind, placebo-controlled trial. Neonatology. 2012;102(3):178-184.

14. Jiménez E, et al. Isolation of commensal bacteria from umbilical cord blood of healthy neonates born by cesarean section. Curr Microbiol. 2005;51(4):270-274

15. Perez PF, et al. Bacterial imprinting of the neonatal immune system: lessons from maternal cells? Pediatrics. 2007;119(3):e724-e732.

16. Lauder AP, et al. Comparison of placenta samples with contamination controls does not provide evidence for a distinct placenta microbiota. Microbiome. 2016;4(1):29.

17. Seferovic MD, et al. Visualization of microbes by $16 \mathrm{~S}$ in situ hybridization in term and preterm placentas without intraamniotic infection. Am J Obstet Gynecol. 2019;221(2):146.e1-146.e23.

18. Martinez KA, et al. Bacterial DNA is present in the fetal intestine and overlaps with that in the placenta in mice. PLoS One. 2018;13(5):e0197439.

19. Salter SJ, et al. Reagent and laboratory contamination can critically impact sequence-based microbiome analyses. BMC Biol. 2014;12:87.

20. Leiby JS, et al. Lack of detection of a human placenta microbiome in samples from preterm and term deliveries. Microbiome. 2018;6(1):196

21. Theis KR, et al. Does the human placenta delivered at term have a microbiota? Results of cultivation, quantitative real-time PCR, 16S rRNA gene sequencing, and metagenomics. Am J Obstet Gynecol. 2019;220(3):267.e1-267.e39.

22. de Goffau MC, et al. Human placenta has no microbiome but can contain potential pathogens. Nature. 2019;572(7769):329-334.

23. Stinson LF, Boyce MC, Payne MS, Keelan JA. The not-so-sterile womb: evidence that the human fetus is exposed to bacteria prior to birth. Front Microbiol. 2019;10:1124.

24. Stout MJ, et al. Identification of intracellular bacteria in the basal plate of the human placenta in term and preterm gestations. Am J Obstet Gynecol. 2013;208(3):226.e1-226.e7.

25. Callahan BJ, McMurdie PJ, Rosen MJ, Han AW, Johnson AJ, Holmes SP. DADA2: high-resolution sample inference from Illumina amplicon data. Nat Methods. 2016;13(7):581-583.

26. Knights D, et al. Bayesian community-wide culture-independent microbial source tracking. Nat Methods. 2011;8(9):761-763.

27. Balsara ZR, Ross SS, Dolber PC, Wiener JS, Tang Y, Seed PC. Enhanced susceptibility to urinary tract infection in the spinal cord-injured host with neurogenic bladder. Infect Immun. 2013;81(8):3018-3026.

28. Borghi E, et al. Antenatal microbial colonization of mammalian gut. Reprod Sci. 2019;26(8):1045-1053.

29. Li L, Mendis N, Trigui H, Oliver JD, Faucher SP. The importance of the viable but non-culturable state in human bacterial pathogens. Front Microbiol. 2014;5:258.

30. Perez-Muñoz ME, Arrieta MC, Ramer-Tait AE, Walter J. A critical assessment of the "sterile womb" and "in utero colonization" hypotheses: implications for research on the pioneer infant microbiome. Microbiome. 2017;5(1):48.

31. Chu DM, Ma J, Prince AL, Antony KM, Seferovic MD, Aagaard KM. Maturation of the infant microbiome community structure and function across multiple body sites and in relation to mode of delivery. Nat Med. 2017;23(3):314-326.

32. Jiménez E, et al. Is meconium from healthy newborns actually sterile? Res Microbiol. 2008;159(3):187-193.

33. Fichorova RN, et al. Maternal microbe-specific modulation of inflammatory response in extremely low-gestational-age newborns. MBio. 2011;2(1):e00280-e00210.

34. van de Pavert SA, Mebius RE. New insights into the development of lymphoid tissues. Nat Rev Immunol. 2010;10(9):664-674.

35. Hoorweg K, et al. Functional differences between human NKp44(-) and NKp44(+) RORC(+) innate lymphoid cells. Front Immunol. 2012;3:72.

36. Mjösberg JM, et al. Human IL-25- and IL-33-responsive type 2 innate lymphoid cells are defined by expression of CRTH2 and CD161. Nat Immunol. 2011;12(11):1055-1062.

37. Gomez de Agüero M, et al. The maternal microbiota drives early postnatal innate immune development. Science. 2016;351(6279):1296-1302.

38. Li N, et al. Memory CD4 ${ }^{+} \mathrm{T}$ cells are generated in the human fetal intestine. Nat Immunol. 2019;20(3):301-312.

39. Caporaso JG, et al. Ultra-high-throughput microbial community analysis on the Illumina HiSeq and MiSeq platforms. ISME J. 
2012;6(8):1621-1624.

40. Altschul SF, Gish W, Miller W, Myers EW, Lipman DJ. Basic local alignment search tool. J Mol Biol. 1990;215(3):403-410.

41. McMurdie PJ, Holmes S. phyloseq: an R package for reproducible interactive analysis and graphics of microbiome census data. PLoS One. 2013;8(4):e61217.

42. Paulson JN, Stine OC, Bravo HC, Pop M. Differential abundance analysis for microbial marker-gene surveys. Nat Methods. 2013;10(12):1200-1202.

43. Kelly BJ, et al. Power and sample-size estimation for microbiome studies using pairwise distances and PERMANOVA. Bioinformatics. 2015;31(15):2461-2468.

44. Chen J, et al. Associating microbiome composition with environmental covariates using generalized UniFrac distances. Bioinformatics. 2012;28(16):2106-2113. 